\title{
Using Published Medical Results and Non-homogenous Data in Rule Learning
}

\author{
Janusz Wojtusiak \\ George Mason University \\ Fairfax, VA, USA \\ jwojtusi@gmu.edu
}

\author{
Katherine Irvin \\ George Mason University \\ Fairfax, VA, USA \\ kirvin@gmu.edu
}

\author{
Aybike Birerdinc \\ INOVA Health System \\ Fairfax, VA, USA \\ abirerdi@yahoo.com
}

\author{
Ancha V. Baranova \\ George Mason University \\ Fairfax, VA \\ abaranov@gmu.edu
}

\begin{abstract}
Many factors limit researchers from accessing studies' original data sets. As a result, much medical and healthcare research is based off of systematic reviews and meta-analysis of published results. However, when research involves the use of aggregated data from multiple studies, traditional machine learning-based means of analysis cannot be used. This paper describes diversity of data and results available in published manuscripts, and relates them to a rule learning method that can be applied to build classification and predictive models from such input. The method can be used to support meta-analysis and systematic reviews. Two application areas are used to illustrate the discussed issues: diagnosis of liver diseases in patients with metabolic syndrome, and detection of polycystic ovary syndrome.
\end{abstract}

Keywords- Aggregated data, Meta-analysis, Published result, Rule learning, Systematic reviews

\section{INTRODUCTION}

Systematic reviews along with secondary data analysis are an important way of gaining medical and healthcare knowledge. They often require significantly less resources than performing randomized clinical trials. The goal of preforming systematic reviews is to bring together evidence from multiple published studies (often contradictory) and arrive at general conclusions. Large groups of people within the Cochrane Collaboration, Campbell Collaboration, and similar collaboratives use strict methodologies and guidelines in performing systematic reviews. This is because the original data used in studies are rarely available due to patient privacy, confidentiality, and reluctance for data sharing among research groups [17], and only the contents of the published work are available.

Despite the significant growth of machine learning, methods developed have not traditionally been used to support systematic reviews. One important reason for this is that the majority of machine learning algorithms are designed to work with data on an individual level (i.e. representing individual patients), but not with summaries, results, and aggregates in various forms (like those used in systematic reviews). Therefore, the analysis of published results and data requires a new specialized class of machine learning methods able to deal with non-homogenous inputs.
The goal of the presented research is to develop a methodology for building classification or predictive models from results and aggregated data present in published papers. It is sufficient that these papers contain aggregated data and/or results related to the problem.

This paper aims at presenting different forms of data within healthcare publications, and discusses challenges related to learning from them. It also briefly discusses modifications to the AQ21 rule learning system that enables learning from several of the discussed forms of data. Although the focus of this paper is on machine learning of attributional rules, and in particular the AQ21 system, most of the presented methods are applicable to other knowledge representations (decision trees, Bayesian networks, etc.).

Two applications of the AQ21 system on aggregated clinical data collected from studies on patients with metabolic syndrome (MS) and polycystic ovary syndrome (PCOS) are presented in this paper. The areas were chosen for both methodological and medical reasons. The methodological reason being that numerous publications are available in these two areas making it relatively easy to access. The medical reason being that not only do these conditions affect very large populations of patients, but there is no consensus on their causes and the best practices.

The central objective of this paper is to summarize types and forms of data that can be derived from publications, as well as present how machine learning can be used to analyze the data. It is not the intention of this paper to discuss how the data can be automatically derived from publications, nor how machine learning can help in selecting relevant publications for systematic reviews.

\section{RESUlTS AS DATA}

One of the key challenges in using machine learning to analyze results is the diversity of the published studies. Publications include tables that summarize data, figures illustrating trends among cohorts of patients, correlation coefficients among selected (usually not all) variables, study inclusion criteria, definitions, and others. These data are very different from individual examples for which machine learning methods are designed to work with. This section, illustrated by MS and PCOS applications, briefly characterizes types of data/information/knowledge present in healthcare publications, and presents the process of using them to prepare the input data for machine learning. 


\section{A. Literature selection}

Published literature is represented in a wide array of different research studies including clinical trials, observational studies, and comparative effectiveness studies. Having different types of research studies creates two major distinctions between the data obtained from the studies - the type of data (qualitative/quantitative or baseline/outcome) and the number of cohorts, participants, and data variables obtained from each. Additionally, it is important to consider how the data were collected (i.e., through randomized trial, observationally, or existing medical records) which affects the reliability and the biases within the data.

The selection of works to be used is a widely discussed issue [7], which applies to all systematic reviews and metaanalyses. It is usually conducted by a panel of experts and is based on criteria such as relevance of studies, quality of publications, cohort sizes, and others.

For example, of the twenty-five PCOS-related papers analyzed, eight of them could be classified as comparative effectiveness studies that compared drug treatments, tests, surgeries, environmental/genetic factors, or life style changes in correlation with PCOS. Another eleven of the papers could be identified as clinical trials. These contained the most significant amounts of data used in the analysis in terms of the number of patient cohorts and measured attributes. The majority of the papers included information identifying and addressing PCOS as well as the medical developments within treatment. However, six of the twenty-five papers were eliminated during the prepping of data for machine learning because they contained no relevant qualitative or quantitative variable values that could be used.

\section{B. What is included in publications}

There is no standardization in what data are included in publications and in what forms. Although some journals require structured manuscripts with specific sections, there are no standard formats in which data and results are presented. This is mainly because it is not possible to create one standard template that fits the wide range of topics covered by journals. The data and results generally come in tables, plots (that are usually equivalent to tables), and as a free text. Preparing them for machine learning-ready is time consuming, and requires expertise in the subject and knowledge of machine learning methods.

One challenge faced when applying machine learning to published data is in developing common representation of data and results. For example, there may be available aggregated data tables and inclusion criteria for twenty patient cohorts, two predictive models, and a small individual patient dataset. Each provides unique inputs to the learning problem and should not be disregarded. Results and data include quantitative and qualitative information definitions, individual patient data, aggregated patient data, inclusion criteria, predictive/classification models, correlation coefficients, and statistical significances.

\section{1) Definitions}

Often there is no consensus on the definitions of symptoms and diseases. For example, there is no one consistently used definition of MS - it is usually defined as a combination of obesity, insulin-resistance, hypertension, elevated triglycerides, as well as a decreased level of high-density lipoprotein cholesterol ("good cholesterol"). Similar situation applies to PCOS. Specific definitions of syndromes, diseases, and their variations need to be included in data, as illustrated in Table 1 for the PCOS case.

Table 1: Excerpt of article description \& PCOS definitions

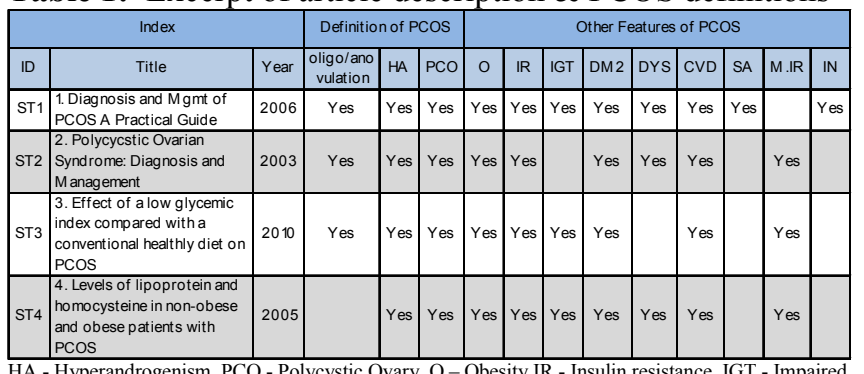

HA - Hyperandrogenism, PCO - Polycystic Ovary, O - Obesity IR - Insulin resistance, IGT - Impaire Glucose Tolerance, DM2 - Diabetes Mellitus 2, DYS - Dyslipidemia, CVD - Cardiovascular Disease,

SA - Sleep Apnea, M.IR - Menstrual Irregularity, IN - Infertility

\section{2) Individual patient data}

A majority of machine learning algorithms are designed to work with individual level data points. For the reasons described in the introduction, and additional ones discussed by Wojtusiak et al. [17], such data are often not available for multiple studies. In some situations, however, there may be limited individual datasets accessible for one or few studies, but not for others.

The most common form of individual data is attributevalue pairs. Because each patient is often described using the same set of attributes, the actual input data is in the form of a flat data table (it is important to note that some machine learning methods can deal with relational data [13], and predicates [6]). The usual situation is that each patient is an individual data point described using a set of attributes $A_{l} \ldots$ $A_{k}$. Typical machine learning programs use such individual data points in the form of attribute-value examples (1) where $v_{l}, v_{2}, \ldots, v_{k}$ are values of attributes $A_{l}, \ldots A_{k}$.

$$
\left(v_{1}, v_{2}, \ldots, v_{k}\right)
$$

Each example is described using the same attributes, thus the input dataset used for learning is in the form of a flat attribute-value table. In the case when some attributes are not present in a description of a specific example, metavalues (a.k.a. missing values) can be used. This form of data is, however, almost never included in published papers for reasons outlined in the introduction.

In the presented PCOS experimental results, no individual patient datum were used to create models (no such datum was available). Conversely, models for predicting MS liver complications were consequently tested for accuracy on an individual patients' dataset. 


\section{3) Aggregated patient data (tables and figures)}

Aggregated data are the most common way of describing cohorts of patients [16][17]. Aggregated values of attributes are given in the form of pairs $\left(\mu_{A}, \sigma_{A}\right)$, where $A$ is a measured attribute, and $\mu_{A}$ and $\sigma_{A}$ denote its mean and standard deviation measured over a group, for which the aggregation was done. Given that means and standard deviations for several parameters are available, each group can be described by an aggregated example given as (2).

$$
\left(\left(\mu_{A_{1}}, \sigma_{A_{1}}\right),\left(\mu_{A_{2}}, \sigma_{A_{2}}\right), \ldots,\left(\mu_{A_{k}}, \sigma_{A_{k}}\right)\right)
$$

For non-numerical attributes, a typically used aggregated form lists the frequencies of values in a group, explicitly showing the distribution of examples. For example, a group of patients may include $70 \%$ White $17 \%$ Black, 2\% Asian patients, and so on.

Aggregated data presents a significant challenge when using machine learning to analyze published results [16][17].

Table 2: Excerpt of aggregated data from PCOS study.

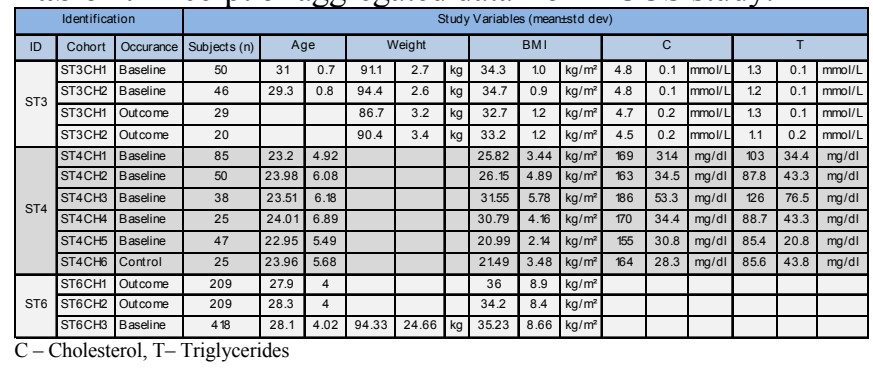

Of the twenty-five PCOS-related papers analyzed eighty-three different cohorts were identified. Seventy-two different qualitative and quantitative variables were identified and organized into one table, whose excerpt is presented in Table 2 above. The table contained qualitative data associated to study participant's characteristics and treatment. The table also contained quantitative data associated with study participants physiological and biochemical states.

\section{4) Inclusion criteria}

Another type of data that describes entire groups in medical or social publications is inclusion criteria for a specific study. It provides important facts about study's participants. Often exclusion criteria are provided instead of explicit inclusion criteria. Aggregated attributes, apply to all individual patients in the described groups. For, example if a study is performed among white males, then each individual subject in the data has precisely these values for attributes describing ethnicity and gender. This is in contrast to aggregated attributes outlined in the previous section. Table 3 depicts part of the inclusion criteria in the PCOS studies.

In contrast to traditional meta-analysis, in the presented method inclusion criteria do not need to match between studies, but rather need to be explicitly listed and are used as part of the input in the learning program.
Table 3: PCOD study inclusion/exclusion criteria excerpt.

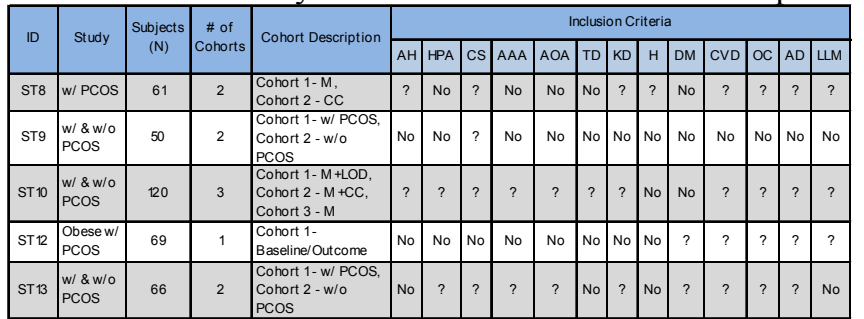

M - Metaformin, CC - Clomiphene Citrate, LOD - Laparoscopic Ovarian Drilling, P- Pioglitazone, AH - Adrenal Hyperplasia, HPA - Hyperprolactinemia, CS - Cushing's syndrome, AA - Acromegaly Adrenal Androgen-producing Tumors, AOA- Acromegaly Ovarian Androgen-producing Tumors, TD - Thyroid disorder, KD - Kidney Dysfunction, H - Hypertension, DM - Diabetes Mellitus, CVD Cardiovascular Disease, OC - Oral Contraceptives, AD - Anti-Depressants, LLM - Lipid-Lowering Medication

\section{5) Predictive/classification models}

Published results may include existing classification or predictive models related to the learning task. Although complete models are rarely included, they may be obtained from authors (if not proprietary). Typically various kinds of regression models, decision trees, Bayesian networks, neural networks, and rule-based models are used.

Different approaches can be used in order to incorporate existing models: incremental learning, which requires existing models to be in the same form as the target model; sampling; using existing models to weight examples; and using existing models to label data in "interesting" cases (a form of active learning). In general, the problem of learning from existing models is not properly addressed in ML.

\section{6) Correlations}

Correlation coefficients, often reported in publications, can be used to better estimate quality (based on estimated coverage/accuracy) of a learned hypotheses. Virtually all methods for calculating quality of hypotheses (rules as well as other representations) are based on their coverage. When learning from published results, particularly from aggregated data, the numbers of individual patients satisfying candidate hypotheses (coverage) are not known, and need to be estimated. Additional information in the form of correlation coefficients can be used to better calculate the coverage.

Table 4: Excerpt of PCOD study correlation coefficients.

\begin{tabular}{|c|c|c|c|c|c|c|c|c|c|c|c|}
\hline ID & Cohort & Cohort & \multicolumn{9}{|c|}{ Study Variables Correlation Coefficients } \\
\hline \multirow{2}{*}{ ST9 } & \multirow{2}{*}{$\mathrm{ST} 9 \mathrm{CH} 1$} & \multirow{2}{*}{$\mathrm{ST} 9 \mathrm{CH} 2$} & \multicolumn{3}{|c|}{ Preptin \& Fasting Insulin } & \multicolumn{3}{|c|}{ Preptin \& HOM A-IR } & \multicolumn{3}{|c|}{$\begin{array}{c}\text { Preptin \& Ferriman-Gallwey } \\
\text { Score }\end{array}$} \\
\hline & & & $\mathrm{r} \mathrm{r}=0.323$ & $\mathrm{p}$ & $<0.022$ & $r=0.319$ & & $<0.024$ & $r=0.689$ & & $<0.001$ \\
\hline & & & \multicolumn{3}{|c|}{ FSH\& Fasting Insulin } & \multicolumn{3}{|c|}{ FSH\& HOM A-IR } & \multicolumn{3}{|c|}{ Adiponectin \& FSH } \\
\hline \multirow{2}{*}{ ST 14} & ST14CH1 & & $\begin{aligned} \mathrm{r} & =0.41\end{aligned}$ & $p$ & $=0.01$ & $\mathrm{r}=0.33$ & $\mathrm{p}$ & $=0.03$ & $\mathrm{r}=0.4$ & $p$ & $=0.01$ \\
\hline & $\mathrm{ST} 14 \mathrm{CH} 3$ & & $\begin{aligned} \mathrm{r} & =0.59\end{aligned}$ & $\mathrm{p}$ & $=0.01$ & $=0.54$ & $\mathrm{p}$ & $=0.01$ & $=0.67$ & $p$ & $=0.005$ \\
\hline \multirow{2}{*}{ ST16 } & & & \multicolumn{3}{|c|}{ BMI\&Adiponectin } & \multicolumn{3}{|c|}{ HOMA-IR \& Adiponectin } & \multicolumn{3}{|c|}{ Insulin \& Adiponectin } \\
\hline & & & $\begin{aligned} &=-0.654 \\
&\end{aligned}$ & $p$ & $<0.005$ & $r=-0.615$ & $p$ & $<0.005$ & $\begin{aligned} &=-0.639 \\
&\end{aligned}$ & & $<0.005$ \\
\hline \multirow{2}{*}{ ST23 } & \multirow{2}{*}{ ST23CHR-B } & \multirow{2}{*}{ ST23CH2-O } & \multicolumn{3}{|c|}{\begin{tabular}{|c|c|}
1 & $1 / 1$ \\
Omentin-1\&BMI
\end{tabular}} & \multicolumn{3}{|c|}{ Omentin-1\&WHR } & \multicolumn{3}{|c|}{ Omentin-1\& Glucose } \\
\hline & & & \multicolumn{3}{|c|}{$T=1-0.4 \quad|D|=10.072$} & \multicolumn{3}{|l|}{$r=-0.445$} & \multicolumn{3}{|c|}{\begin{tabular}{|l|l|l|l|l|}
$\mathrm{r}$ & $=-0.471$ & $\mathrm{p}$ & $=0.031$ \\
\end{tabular}} \\
\hline
\end{tabular}

7) Statistical significance (p-values)

Statistical significance usually given as p-values is another form of data present in publications. It can be used to weight data points and attributes when learning. Cohorts of patients and attributes from studies whose results are significant are more important for learning, thus are weighted higher. 
Table 5: Excerpt of PCOD study statistical significance.

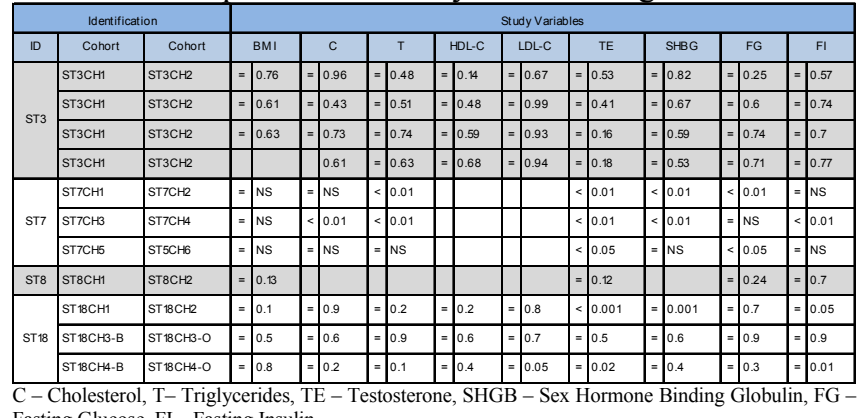

Fasting Glucose, FI - Fasting Insulin

\section{Preparation of data for rule learning}

Before data can be prepared for input, it must be collected from the selected publications and organized into a relational database. The database consists of the aforementioned tables with definitions, inclusion criteria, aggregated patient data, correlation coefficients, and other statistical results (Table 6).

The majority of machine learning software, including AQ21 used in this study, takes input data in the form of data tables in which each example is represented using equal number of attributes. The major difference between AQ21 and other rule learning software is that it can use aggregated and individual examples. Because of the variance in what attributes are reported in different publications, missing values need to be extensively used (note the semantic distinction between unknown, not-applicable and irrelevant missing values described by Michalski and Wojtusiak [9]). For example, out of 152 attributes collected in the MS study, only one was reported in all publications. Similarly, in the PCOS study, about $78 \%$ of data values were missing - many of the attributes were present in only a single study.

Results are also reported in different units among the various studies. Appropriate conversions need to be done.

Finally, all data are checked for consistency. This includes checking for normal or reasonable ranges of values, typos, and variable usefulness for the application at hand. An excerpt of the final data table used is shown in Table 6.

\section{RULE INDUCTION FROM PUBLISHED RESULTS}

\section{A. AQ algorithm}

Many algorithms are available for inducing rules from individual data. Despite their differences, the algorithms have two common elements: rule construction, and rule evaluation. The described method for learning from published results and aggregated data focuses on these two algorithm elements, thus, it is applicable beyond the AQbased systems described here. AQ is a family of rule learning systems, including the newest AQ21 [15], developed in the GMU Machine Learning and Inference Laboratory (www.mli.gmu.edu). AQ21's elements are designed specifically to deal with clinical data. In the core of all AQ systems is a simple version of $\mathrm{A}^{\mathrm{q}}$ algorithm for generating attributional rules [8][15][17]. Attributional rules are more expressive than typical $I F \ldots T H E N \ldots$. rules because they may include a variety of constructs, including internal conjunction and disjunction, comparison of attributes, counting attributes, simple arithmetic expressions, exceptions, preconditions, and many others [8]. Rule learning usually results in more than one rule in the systems output (rulesets and ruleset families) - these rules usually should be interpreted together).

The AQ learning works in two main stages: rule construction and rule optimization. At the core of the first stage is a star generation algorithm, which creates multiple generalizations (in the form of attributional rules), called stars, of a selected positive example. These rules do not cover negative examples (except for noise). A combination of rules selected from one or more stars is used as a generated hypothesis. More detailed description of the AQ21 system is available in another ICMLA 2011 paper [18].

The rule generation stage can be extended to deal with aggregated examples and published results. This is done by operators that combine the logic of rule learning with statistical information derived from publications [16][17].

The most important issue within rule learning is the evaluation/estimation of coverage. Rule selection and optimization criteria are, to a large extent, based on how

Table 6: Except of PCOD standardized data ready for AQ21 rule learning.

\begin{tabular}{|c|c|c|c|c|c|c|c|c|c|c|}
\hline \multicolumn{3}{|c|}{ Identification } & \multicolumn{8}{|c|}{ Study Variables (mean \pm std dev) } \\
\hline Cohort & Occurance & PCOS & Weight & $\operatorname{BMI}\left(\mathrm{kg} / \mathrm{m}^{2}\right)$ & $\mathrm{TE}(\mathrm{ng} / \mathrm{dL})$ & SHGB (ug/mL) & $\mathrm{FG}(\mathrm{mg} / \mathrm{dL})$ & $\mathrm{FI}$ (ulU/mL) & FGS & $E(p g / m L)$ \\
\hline ST6CH1 & Outcome & Yes & ? & $36+/-8.9$ & $61.3+/-32$ & $3.4+/-18.7$ & $89.2+/-16.5$ & $22.6+/-20.7$ & $14.7+1-8.2$ & $?$ \\
\hline $\mathrm{ST} 6 \mathrm{CH} 2$ & Outcome & Yes & $?$ & $34.2+/-8.4$ & $63.1+/-28.4$ & $3.6+/-20.3$ & $88.9+/-18.6$ & $22.4+/-30$ & $14.4+/-7.4$ & $?$ \\
\hline $\mathrm{ST} 6 \mathrm{CH} 3$ & Baseline & Yes & $94.33+/-24.66$ & $35.23+/-8.66$ & $62.03+/-28.63$ & $3.4+/-18.0$ & $?$ & $22.99+/-26.63$ & $14.4+/-7.88$ & $?$ \\
\hline ST8CH1 & Baseline & Yes & $?$ & $29.7+/-6.13$ & $119+/-84$ & $?$ & $91.71+/-9.45$ & $15.62+/-18.13$ & $12.67+/-7.66$ & $49.59+/-25.37$ \\
\hline ST8CH2 & Baseline & Yes & $?$ & $27.3+/-5.9$ & $86+/-44$ & $?$ & $86.58+/-13.24$ & $8.77+/-5.43$ & $9.71+/-6.19$ & $38.73+/-17.02$ \\
\hline ST9CH1 & Baseline & Yes & $53.04+/-5.32$ & $22.53+/-5.31$ & $62.79+/-27.13$ & $5.2+/-5.0$ & $90.84+/-11.14$ & $20.55+/-13.4$ & $15.4+/-5.16$ & $42.01+/-16$ \\
\hline ST9CH2 & Control & Yes & $54.33+1-12.3$ & $20.54+/-1.95$ & $49.06+/-16.8$ & $6.1+1-2.0$ & $90.96+/-14.88$ & $12.6+/-5.92$ & $9+/-2.41$ & $109+/-85.8$ \\
\hline ST19CH1 & Control & Yes & $80.5+/-4.3$ & $30+/-0.6$ & $37.4+/-5.7$ & $7.6+/-0.5$ & $82.8+/-3.5$ & $7.2+/-3$ & $4.8+/-1.2$ & $?$ \\
\hline ST19CH2 & Baseline & Yes & $80.2+/-3.7$ & $30.7+/-1.2$ & $103.7+/-11.5$ & $4.9+/-0.5$ & $97+/-9.6$ & $21.3+/-5.9$ & $15+/-1.9$ & $?$ \\
\hline ST19CH2 & Outcome & Yes & $79.6+/-8.4$ & $?$ & $92.2+/-14.4$ & $6.2+/-1.3$ & $85.4+/-6.1$ & $11.6+/-1.4$ & $16+/-3$ & $?$ \\
\hline ST22CH1 & Outcome & Yes & $?$ & $30.2+/-1.6$ & $75+1-49$ & $?$ & $?$ & $19.4+1-2.9$ & $21.5+/-1.5$ & $42.1+/-5.8$ \\
\hline ST22CH1 & Outcome & Yes & $?$ & $28.5+/-1.8$ & $57.2+/-32$ & $?$ & $?$ & $13.8+/-1.9$ & $14.6+1-0.7$ & $66.4+/-11.1$ \\
\hline
\end{tabular}

TE - Testosterone, SHGB - Sex Hormone Binding Globulin, FG - Fasting Glucose, FI - Fasting Insulin , E - Estradiol 
A related field called Literature-based discovery (LBD) concerns methods for identifying unknown relationships in data drawn from published results [5]. By bringing together results published in several papers, new relationships that were not considered in the original studies can be found. While the general framework of LBD is somewhat similar to the described rule learning method, its goal is to discover relationships, rather than build models.

\section{Discussion AND CONCLUSION}

Published medical studies include a wide range of data that is not properly utilized by traditional systematic reviews. The described methodology for analyzing published results is intended to complement currently used techniques in systematic reviews and meta-analyses. With rapidly changing clinical knowledge, automated methods with the ability to incrementally update knowledge may prove to be the needed method to keep reviews up to date. This is particularly important because evidence-based medicine requires clinicians to access the newest results.

Preliminary applications of the method in the two areas, has shown that it is capable of producing simple rules that can aid physicians in diagnostic tasks. Though the method seems promising, further work is needed to accurately test and implement the created rules in clinical practice.

While the research on the methodology is still in progress, the envisioned extension of the AQ21 system will allow for the use of all types of data and results mentioned in this paper. Currently, AQ21 supports individual-level and aggregated data, and weights of attributes and examples. The system is envisioned to be a platform for human-oriented machine learning in medical and healthcare applications.

Systematic investigation and testing of the described methods is beyond scope of the short conference paper. However, we are in the process of using Monte Carlo simulation methods to prepare large number of simulated datasets that can be used to test the methods in diverse number of situations. In addition to the experimental testing, theoretical investigation of the method is being prepared.

\section{ACKNOWLEDGMENTS}

The presented research has been conducted in part in the GMU Machine Learning and Inference Laboratory whose activities are supported by the National Institute of Standards and Technology and Mason-Inova fund grants.

\section{REFERENCES}

[1] The Cochrane Collaboration, The Cochrane Manual 4, 2008. [updated 14 August 2008].

[2] Cohen, A.M., Ambert, K., McDonagh, M., "Cross-Topic Learning for Work Prioritization in Systematic Review Creation and Update" JAMIA, 16, 5, 690-704, 2009.
[3] Davies, F., Boruch, R., "The Campbell Collaboration Does for Public Policy what Cochrane Does for Health," BMJ, 323, 294-295, 2001.

[4] DHQP. "FAQs - Polycystic Ovary Syndrome (PCOS)." Women's Health - PCOS Fact Sheet, U.S. DHHS, Office on Women's Health, 17 Mar. 2010. Web. 17 July 2011 $<$ http://www.womenshealth.gov/publications/ourpublications/fact-sheet/polycystic-ovary-syndrome.pdf $>$.

[5] Gordon, M., Lindsay, R.K., Fan, W., "Literature-Based Discovery on the World Wide Web," ACM TOIT, 2(4), 261275, 2002.

[6] Lavrac N., \& Dzeroski, S., Inductive Logic Programming: Techniques and Applications, Ellis Horwood, NY, 1994.

[7] Lipsey M.W., Wilson, D., Practical Meta-analysis, Sage Publications, Inc., 2000.

[8] Michalski, R. S., "ATTRIBUTIONAL CALCULUS: A Logic and Representation Language for Natural Induction," Reports of the MLI Laboratory, MLI 04-2, George Mason University, Fairfax, VA, April, 2004.

[9] Michalski, R. S. \& Wojtusiak, J., "Reasoning with Missing, Not-applicable and Irrelevant Meta-values in Concept Learning and Pattern Discovery," Technical Report 2005-02, Collaborative Research Center 637, University of Bremen, Germany, 2005.

[10] Michalski, R. S. \& Wojtusiak, J., "The Distribution Approximation Approach to Learning from Aggregated Data," Reports of the MLI Laboratory, MLI 08-2, George Mason University, Fairfax, VA, 2008.

[11] Sciarretta, S., Palano, F., Tocci, G., Baldini, R., Volpe, M., "Antihypertensive Treatment and Development of Heart Failure in Hypertension: A Bayesian Network Meta-analysis of Studies in Patients With Hypertension and High Cardiovascular Risk," Archives of Internal Medicine, 171(5), 384-394, 2011.

[12] Sutton, A.J., Abrams, K.R., "Bayesian methods in metaanalysis and evidence synthesis," Statystical Methods in Medical Research, 10, 277-303, 2001.

[13] De Raedt, L., Logical and Relational Learning, SpringerVerlag, 2008.

[14] Wojtusiak, J., "AQ21 User's Guide," Reports of the MLI Laboratory, MLI 04-3, George Mason University, Fairfax, VA, September, 2004.

[15] Wojtusiak, J., Michalski, R. S., Kaufman, K. \& Pietrzykowski, J., "The AQ21 Natural Induction Program for Pattern Discovery: Initial Version and its Novel Features," The 18th IEEE ICTAI, Washington D.C., (2006).

[16] Wojtusiak, J., Michalski, R. S., Simanivanh, T. \& Baranova, A. V., "Towards application of rule learning to the metaanalysis of clinical data: An example of the metabolic syndrome," IJMI, 78, 12, e104-e111, 2009.

[17] Wojtusiak, J., Baranova, A.V., "Model Learning from Published Aggregated Data" Learning Structure and Schemas from Documents, M. Biba and F. Xhafa (Eds.) Springer, 2011.

[18] Wojtusiak, J., Ngufor, C., Shiver, J., Ewald, R., "Rule-based Prediction of Medical Claims' Payments: A Method and Initial Application to Medicaid Data" ICMLA 2011. 